\title{
Giant Kerr Rotation Enhancement in Magneto-plasmonic Metamaterials
}

\author{
E. Atmatzakis ${ }^{1}$, N. Papasimakis ${ }^{1}$, V. Fedotov ${ }^{1}$ and N. I. Zheludev ${ }^{1,2}$ \\ 1. Optoelectronics Research Centre and Centre for Photonic Metamaterials, University of Southampton, Southampton SO17 1BJ, \\ United Kingdom, \\ 2. Centre for Disruptive Photonic Technologies, Nanyang Technological University, Singapore
}

\begin{abstract}
We report for the first time an order of magnitude enhancement of Kerr rotation in hybrid plasmonic/ferromagnetic metamaterial resonators. Our results pave the way towards magnetically controlled metamaterials and integrated magneto-plasmonics.

OCIS codes: (190.3270) Kerr effect; (160.3918) Metamaterials
\end{abstract}

Interfacing plasmonic and magneto-optical materials has recently emerged as a promising approach towards active nanoscale optical elements with significant technological implications: optical isolators, modulators, data storage, sensing, imaging, therapy. Such hybrid systems combine the strong local fields at the plasmonic resonance with the magneto-optical response of ferromagnetic layers leading to magnetic control of the plasmonic response or vice-versa [1,2]. Here we demonstrate direct integration of magneto-optical materials in metamaterial resonators leading to an order of magnitude enhancement of the polar magneto-optical Kerr effect (MOKE).

The system under study is a periodic array of bi-metallic Au/Ni plasmonic ring resonators, where the gold part provides a strong plasmonic response, while the nickel section is responsible for the magneto-optical effects. Taking into account the high losses of Ni which damp the plasmonic response of the resonators, as well as current manufacturing and measuring limitations, we fix the composition of the rings to $3 / 4 \mathrm{Au}$ and $1 / 4 \mathrm{Ni}$ (see Fig. 1a). The sample was fabricated by a multi-step procedure comprising e-beam lithography, film evaporation, and lift-off processes. The manufactured sample consists of bi-metallic rings with a mean diameter of $200 \mathrm{~nm}$ and a cross-section of $50 \times 50 \mathrm{~nm}^{2}$. The unit cell is $400 \times 400 \mathrm{~nm}^{2}$, while the overall size of the array is $100 \times 100$ $\mu \mathrm{m}^{2}$.
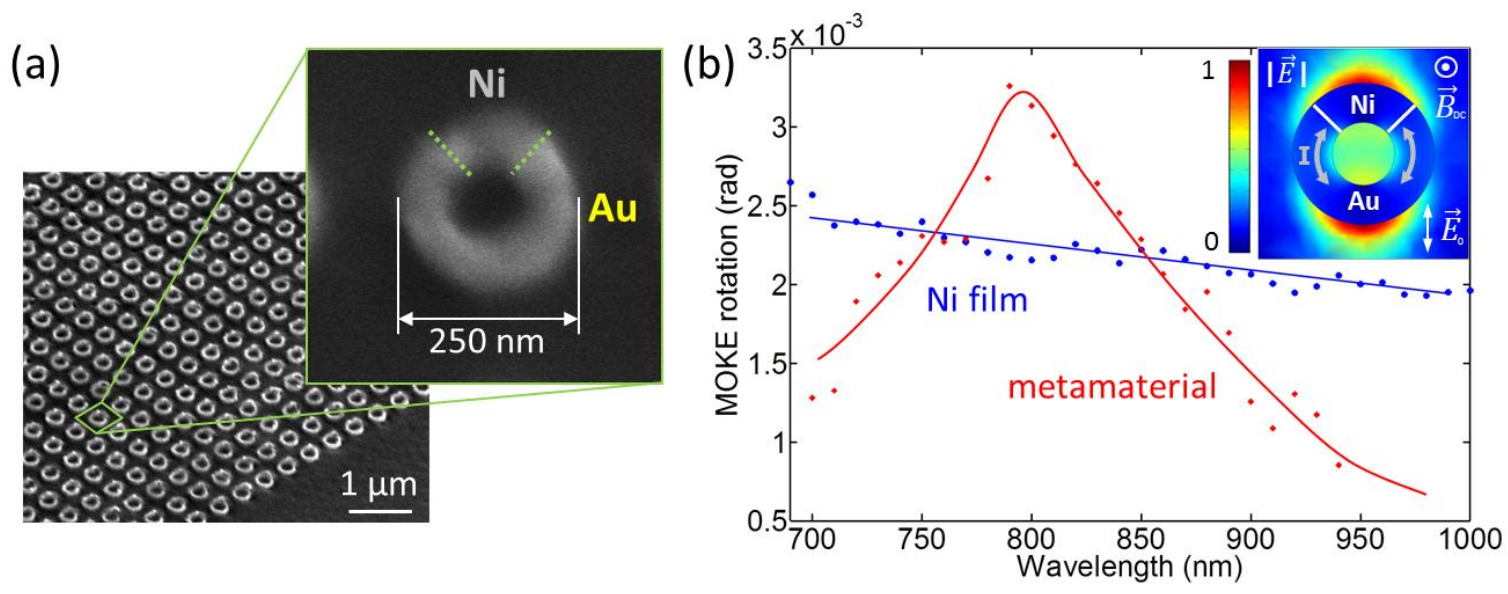

Figure 1: (a) Scanning electron microscope images of the fabricated metamaterial array and its unit cell. Dashed lines in the inset mark the Au/Ni junctions. (b) Polarization azimuth rotation spectra due to the polar magneto-optical Kerr effect in the presence of a $B_{D C}=200 \mathrm{mT}$ external magnetic field. Blue points correspond to a $50 \mathrm{~nm}$ thick Ni film and red to the bimetallic metamaterial array. Lines serve as guides for the eye. Inset: Numerically simulated electric field map of the metamaterial unit cell at resonance. 
For the measurement of the MOKE-induced polarization azimuth rotation, a reflection polarimeter setup was implemented. The output of a Ti-sapphire is modulated by a Faraday modulator (FM) before reaching the sample. The polarization states of the reflected beam are separated by a birefringent prism and only the orthogonal to the incident is arriving at the detector. The signal reaching the detector consists of two components. The FM signal appears as the second harmonic of the modulated frequency whereas the MOKE signal is the fundamental frequency component. A lock-in detection allows for the separation of the two. A static magnetic field generated by strong Neodymium magnets, is responsible for the creation of the MOKE.

The measurement was performed using a continuous $\mathrm{Ni}$ film of $50 \mathrm{~nm}$ thickness and a metamaterial array as samples. A $200 \mathrm{mT}$ static magnetic field was applied normal to the plane of the samples (see inset to Fig. 1b) and the resulting Kerr rotation was measured close to normal incidence. The corresponding MOKE spectra are presented in Fig. 1b. The response of the Ni film is approximately flat and decreases with increasing wavelength as it corresponds to the typical behavior of $\mathrm{Ni}$ at that wavelength range. On the other hand, the metamaterial array exhibits resonant rotation at around $800 \mathrm{~nm}$ with a FWHM of approximately $300 \mathrm{~nm}$. At the peak of the resonance, the metamaterial sample presents a rotation of $3.2 \mathrm{mrad}, 1.5$ times higher rotation compared to the film. Moreover, considering that the ferromagnetic material covers only a small fraction (5\%) of the metamaterial array, the rotation enhancement normalized to the Ni filling factor is about 30 .

The rotation maximum correlates with the plasmonic resonance of the bimetallic rings indicating that the origin of the observed MOKE enhancement lies in the strong plasmonic fields at the Ni section of the rings. In particular, at the wavelength range of interest, the bimetallic rings support a symmetric dipole excitation, where in phase currents flow along the two halves of the ring (see inset to Fig. 1b). As a result charge accumulates at the $\mathrm{Au} / \mathrm{Ni}$ junctions, consequently leading to field concentration at the $\mathrm{Ni}$ section and a substantial enhancement of the observed MOKE.

In conclusion, we demonstrate experimentally that bimetallic plasmonic/ferromagnetic metamaterial arrays can enhance substantially the Magneto-optical Kerr Effect. The suggested approach provides an efficient compact platform for enhancement and control of the magneto-optical material properties. A metamaterial array can be integrated in optical devices, providing many levels of control including the wavelength, the magnitude and the phase of the magneto-optical response. We expect that our results will find application in magnetically controlled modulators, optical isolators, magnetic sensing and microscopy.

\section{References}

[1] J. Y. Chin et al., "Nonreciprocal plasmonics enables giant enhancement of thin-film Faraday rotation", Nature Comm. 4, 1599 (2013)

[2] A. Tsiatmas et al., "Optical generation of intense ultrashort magnetic pulses at the nanoscale", New J. Phys. 15, (2013) 